\title{
Learning Style Analytics for Children based on Neurophysiological Algorithmic Model of Affect
}

\author{
${ }^{1}$ Norhaslinda Kamaruddin and ${ }^{2}$ Abdul Wahab Abdul Rahman \\ ${ }^{1}$ Advanced Analytics Engineering Centre, Faculty of Computer and Mathematical Sciences, \\ Universiti Teknologi MARA, 40450 Shah Alam, Selangor Malaysia, norhaslinda@tmsk.uitm.edu.my \\ ${ }^{2}$ Kulliyah of Information and Communication Technology, International Islamic University Malaysia, \\ P.O. Box 10, 50728 Kuala Lumpur, Malaysia, abdulwahab@iium.edu.my
}

\begin{abstract}
Effective learning for children requires the matching of both teaching and learning style in such a way that both teachers and learners can enjoy and absorb the knowledge. Young aged children may not be able to understand the different learning styles, hence, disregard its adoption in learning. Such situation may slow down the children rate of understanding of the learned subject. Moreover, teachers need to manage the learning environment to suit the children learning needs to optimize absorption of information. With variety of education aids that are produced in a fast manner and in a large volume, it is assumed that one technique fits all is impossible to achieved. Such scenario results to the unfulfilled lesson plan objective because each individual has their individual learning style to adapt with the contents. Hence, learning style analytics is required to help teachers to channel their effort for better information dissemination and selection of suitable educational tools. This research focuses on investigating the different characterizations of learning style and suitable learning stimuli to ignite the brain activation function, especially, for children where they may not even understand the different learning styles. It will distinguish the impact of different learning stimuli to the different brain signals reflected by various learning style. The different learning style analytics will help teachers to be able to optimize the learning style for each group of students. The brain activation is mapped onto the affective space model because emotion influences learning motivation. The valence and arousal axes of the affective space model will be plotted and compare with learner's style analytics. The validation of the proposed model is presented by modelling the correlation between learning style and emotion using neurophysiological input from Electroencephalogram (EEG). In the future, the proposed model can be used by learners for self-assessment to reflect continuous learning improvement and for teachers to plan supporting intervention for better teaching pedagogy. This aspiration is in line with the direction of Ministry of Education to transform Malaysia's education in preparation for the future education needs moving forward to industrial revolution (IR4.0).
\end{abstract}

$\underline{\text { Key words: Learning analytics, profiling, electroencephalogram, affective space model, learning style, revolution }}$

\section{INTRODUCTION}

Teachers and students have always been struggling with how to teach and how to learn, respectively. Student has their own learning style and preference. To complicate matters, teachers also have their own teaching style. The matching between learning style and teaching style for every student-teacher in the class is almost impossible and mismatch problem may occur. When the student cannot grasp the content of the subject, student tends to lose interest and start to drop from his/her learning progression. This situation will develop lack of self-confidence, laziness, absenteeism problem and learning avoidance. In fact, 'good teacher' or 'bad teacher may be the product of mismatch between the learning and teaching style rather than the ability of students to learn or the teacher to teach.

Teachers tend to use the same teaching technique for all students to simplify teaching and learning approach (Hamiz et al., 2018). This is because it is hard to streamline teaching approach to multiple ways because the number of students in a class is high. Based on the Ministry of Education (MOE) 2016 report (Anonymous, 2016), there are 4,830,271 students enrolled as

Corresponding Author: Norhaslinda Kamaruddin, Advanced Analytics Engineering Centre, Faculty of Computer and Mathematical Sciences, Universiti Teknologi MARA, 40450 Shah Alam, Selangor, Malaysia, norhaslinda@tmsk.uitm.edu.my 


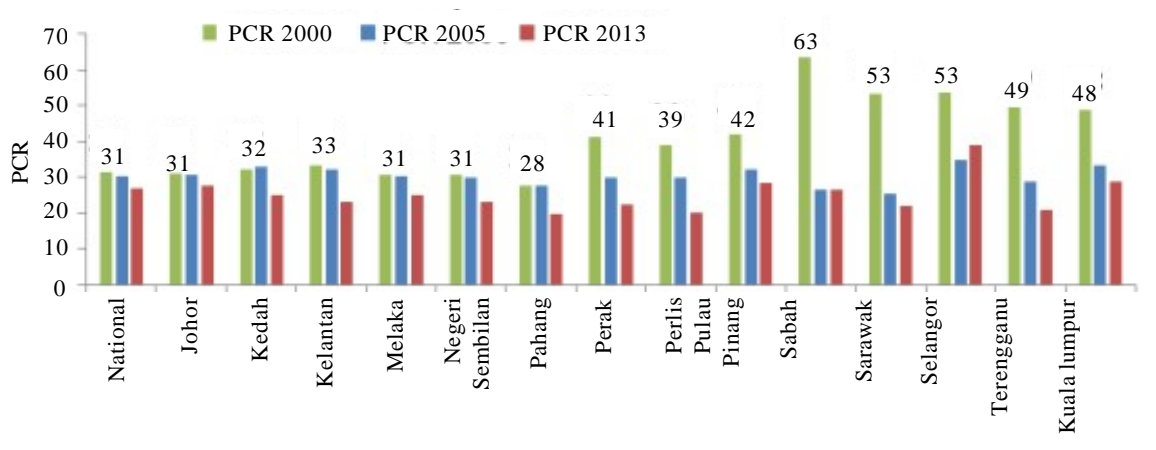

Fig. 1: Pupil to Class Ratio (PCR) for different states in Malaysia

compared to 422,017 teachers from 10,173 schools ranging from preschool to secondary school. Such statistics recorded an approximate school-teacher-student ratio of $1: 41: 475$. At a glance, the ratio indicates that there are 475 students in a school with 41 teachers making it typical for a class to have number of students occupied between 25-45 students. Figure 1 depicts the Pupil to Class Ratio (PCR) for different states ranging from the year of 2000-2013 (UNESCO., 2015). Although, it is obvious that the distribution of PCR is improved, the number is still high. Therefore, it posed as a challenge to the educators to tailor their teaching and learning approach.

Traditionally, learning style identification is conducted using simple questionnaire where the participant can choose the answer from multiple choice questions (Feldman et al., 2015). However, the assessment's result can be nullified by exaggeration or suppression of the learners. Such approach may result to misleading conclusions, if the participants have not answered the test properly and truthfully. The participants may provide false feedback without realizing the relevance of the answer due to learner's lack of motivation to complete the test and their lack of self-awareness of learning preferences, hence, making it difficult to determine the actual personal learning style. An approach to eliminate manipulated answer is needed to get the 'pure' respond from the participants.

In addition, there are no available data using neurophysiological approach to understand learning style. Neurophysiological data collection is not easy and typically only used for medical diagnosis, especially, for brain degenerative diseases such as Parkinson, epilepsy and Alzheimer. With the advancement of technology, it is currently possible to capture brain signal, especially brain activation to further analyze the way brain behaves given different stimuli. Such data collection is significant to garner insights for learning style analytics. Hence, we would like to propose the implementation of Electroencephalogram (EEG), in capturing the neurophysiological signals of the brain wave pattern, to recognize the individual learning style.

Relevant works conducted by previous researchers on learning styles and recognizing it will be discussed in the literature review. Once fundamental understanding is established, we presented our proposed approach using EEG to capture individual learning style. This is because different education stimuli will be differently responded by individual due to his/her learning style. These personalized responses are triggered by brain neurons activation and can be captured and measured using EEG. This is to investigate neurophysiological data garnered from different education stimuli effect based on different learning for developing learning style analytics based on neurophysiological algorithmic model of affect.

Literature review: Psychologist and scientist have developed many models of learning styles and the four well known were the Visual, Aural, Reading/writing and Kinesthetic (VARK) Model (Fleming and Mills, 1992). The VARK Model uses the four sensory modalities that are used for learning information. Visual learners process information better when presented with graphic educational contents, for instance, the use of charts, sketches, diagram and mind maps (Liew et al., 2015). On the other hand, aural/auditory learners prefer listening and interpreting information by the means of pitch, emphasis and speed (Gilakjani and Ahmadi, 2011). These are slightly different from read/write learners who prefer to learn through information presented in written word making it ideal for having printed hands out and textbooks. It is understandable, if they prefer verbal discussion, attending lectures and seek the use of study groups. Subsequently, kinesthetic learner employs a combination of sensory functions that utilize experience to learn, for example, in-class demonstration, hands on exercise and lessons that emphasize on performing an activity such as role-play. Liew et al. (2015) in their experimental result reported that majority of the participants $(81.9 \%)$ had 
unimodal predominant learning style. Such finding is in-line with Fleming (2006) report that majority of the participants who have taken the online test have single learning style preferences (Gilakjani and Ahmadi, 2011). Therefore, it is possible to map the learning style and individual based on his/her response to the educational stimuli.

Human brain function differently and the way each of us capture, process, understand, analyze and retain information/knowledge resulting in a different absorption rate of cognitive content (Willingham et al., 2015). Thus, each individual adopted a different learning style to suit the learner's capabilities, familiarities and behavior towards learning which is very much dependable on the strengths, weaknesses and preferences of the individuals (Feldman et al., 2015). Learning style can also be described as one's ability to gain knowledge and in understanding the learning situations through individual natural and habitual ways (James and Gardner, 1995). Moreover, Fleming and Mills (1992) defined learning style as individual's characteristics and preferred ways of gathering, organizing and thinking about information. Thus, it is reasonable to say that learning style is more of an individual preference for processing information/knowledge in certain types of way but is not a determination of learning ability.

Since, different people have different learning style to process information, it is possible to observe the differences in brain activation. Gevins and Smith (2000) reported that different areas and sides of the brain become active during a specific task based on experimental results recorded from EEG signals from 80 young adults during spatial working memory tasks. Such finding showed that task-related neurophysiological measures could distinguish individuals with relatively high verbal aptitude from those with relatively high nonverbal aptitude based on the utilization of left and right cerebral hemispheres. Chen et al. (2015) performed a simultaneous fNIRS-EEG experiment with visual and auditory stimulation to observe changes in neural activity. The experimental results show that there is a clear distinction between visual and auditory sensory modalities and there is area specificity in brain activation based on the stimuli. Maximal fNIRS responses are captured in visual and auditory area when visual and auditory stimuli presented, respectively. Hence, such experimental result can be extended to different learning style that utilize different sensory perception in brain.

Learning is affected by emotional and motivational factors (Leutner, 2014). The effects are mediated by differences in cognitive processing. Such effect is very much dependent on learner's strengths, weaknesses and preference. If correct learning style is used, the learners may experience positive emotion and on contrary, the learners may feel frustrated and do not wish to pursue learning. Thus, it is hypothesized that there is a strong correlation between emotion and learning style. Moreover, emotion recognition from EEG signals allows the direct assessment of the "inner" state of a user (Jenke et al., 2014) observed that better emotion classification result can be obtained based on brain location over parietal and centro-parietal lobes making it in-line with other findings (Gevins and Smith, 2000; Chen et al., 2015; Wang et al., 2014) reported that using linear dynamic system-based feature smoothing method can significantly improve emotion classification accuracy based on power spectrum feature using visual stimuli. Bhatti et al. (2016) uses audio stimuli to excite brain and elicit emotion. Features from three different domains; namely, time, frequency and wavelet were extracted from recorded EEG signals which were further used by the classifier to recognize human emotions. It was found that EEG is able to classify emotion with comparable accuracy.

\section{MATERIALS AND METHODS}

This research focuses on investigating the different characterizations of learning style and suitable learning stimuli to ignite the brain activation function. It will distinguish the impact of different learning stimuli to the different brain signals reflected by various learning style (Fig. 2). The research methodology adopted is based on

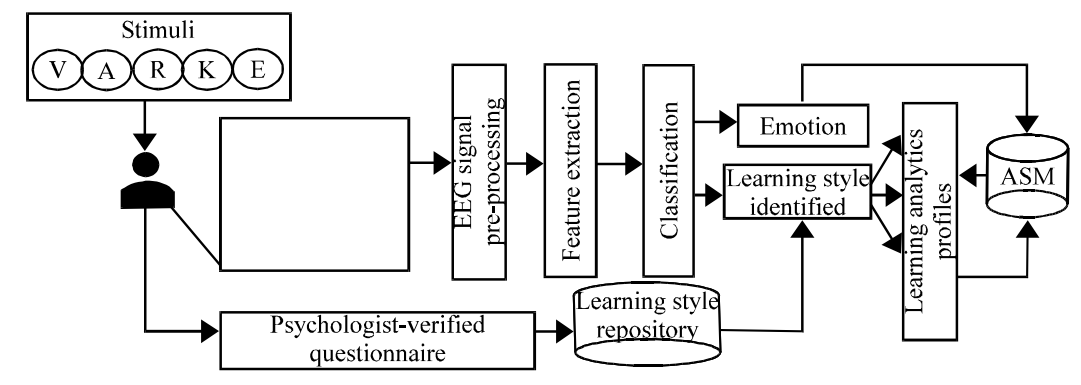

Fig. 2: Conceptual model of Neuro-Physiological Algorithmic Model of Affect Learning Style Analytics (NPAMoA-LSA) 
a fusion of formal, experimental and simulation methods. In this researcher, EEG is used to capture the different brain activation function elicited from different educational stimuli based on hypothesis that different learner styles react differently that excite different parts of the brain. Four different learning stimuli from Aisual (V), Auditory (A), Read/write (R), Kinesthetic (K) will be provided to the participants. In addition, four basic emotions stimuli will also be used to understand individual brain responses to four basic emotions based on the International Affective Pictures (IAPs). Such approach will provide quantitative experimental data for further analysis.

Once the raw EEG data are collected, preprocessing of the EEG signals are needed to ensure the data will be free from artifacts due to eye movements or muscle movement and any other artifacts. Relevant features will be extracted to feed to the classifier for learning style analysis. The results are validated with educationists and learning experts using the subject's answer on the psychologist-verified instruments to get his/her learning style correlation. Further analysis is conducted to match the result obtained from EEG-elicited approach and psychologist-verified instruments. The conceptual model of Neuro-Physiological Algorithmic Model of Affect Learning Style Analytics (NPAMoA-LSA) is presented in Fig. 2.
The EEG signals of each learning type are analyzed and compared. The EEG patterns similarity of different individuals who have the same learning type are measured given the relevant stimuli. Moreover, the differences of the EEG patterns are also captured to compare different learning style. Generalization and specialization of the model are then established. The brain activation is then mapped onto Affective Space Model (ASM) in order to analyze the emotion influence on learning motivation. The valence and arousal axes of the affective space model will be plotted and compare with learner's profiles. The validation of the proposed model is presented by modelling the correlation between learning style and emotion to better understand heutagogy using neurophysiological input (EEG).

\section{RESULTS AND DISCUSSION}

Some preliminary experiments were carried out to understand learning style between normal children and children with learning disabilities based on motor skill learning. In this experiments, 6 participants of normal children and 6 participants with learning disabilities (mainly autistic children) are involved. Figure 3a shows the differences in their arousal responses such that normal children will response to the motor skill learning with active arousal varying from negative to positive
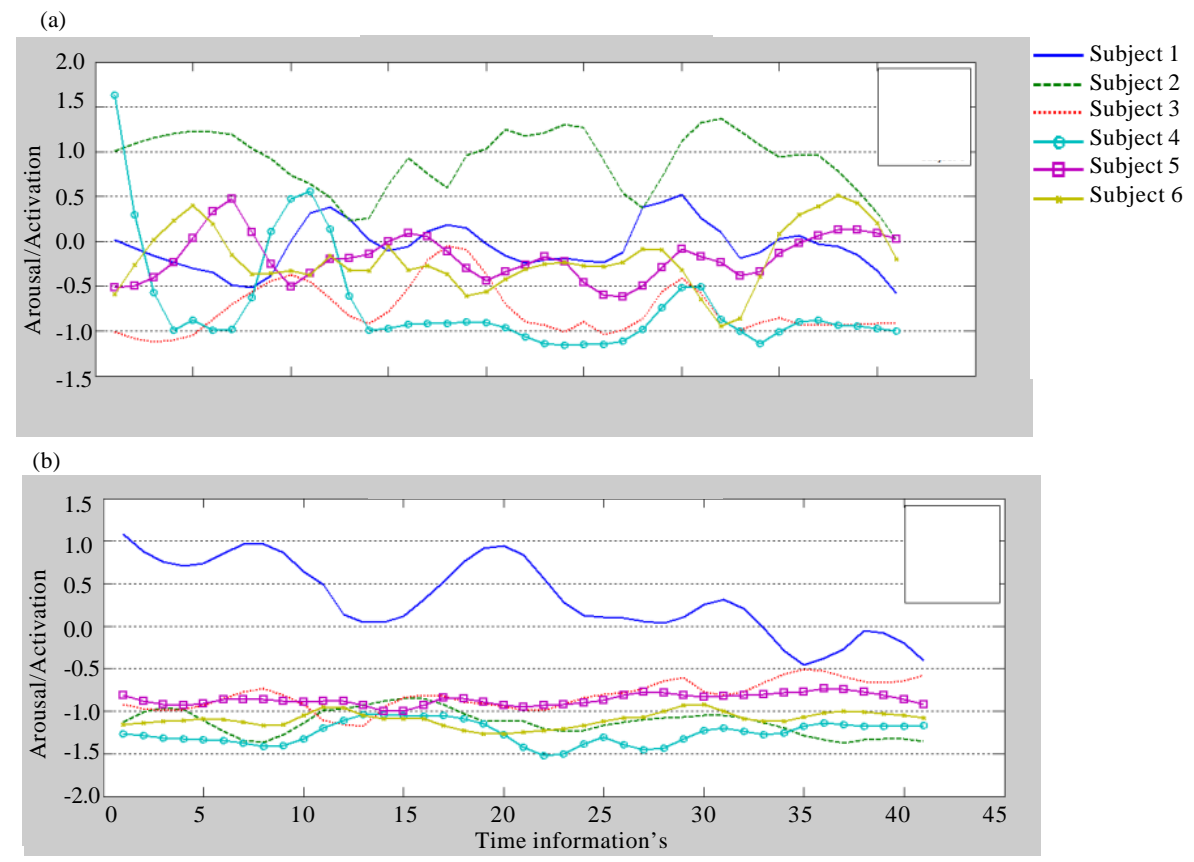

Fig. 3: Preliminary results of motor skill learning differences between: a) Normal children and b) Children with learning disability 
while in Fig. 3b the arousal responses of the children with learning disabilities shows very little changes at negative potential. From the result, it is observed that the proposed model implementation can detect potential of adaptation of children to different stimuli of learning style.

\section{CONCLUSION}

This researcher is still in progress. In this study, we proposed the used of EEG device to capture the neurophysiological signals of the brain wave pattern as input to recognize learner's learning style and further used to develop learning style analytics for generalization purposes. Results shows potential of using the EEG device to identify responses of the brain wave to the motor skill learning as preliminary work. The model is verified with educationists and psychological findings gathered from established instruments. Emotion information yielded from affective space model is also incorporated for model completeness.

\section{RECOMMENDATIONS}

In the future, the proposed model can be used to profile learner for self-determined learning proponents. Learners can benefit from better learning experience using relevant learning aids based on their personalized learning style. The Neuro-Physiological Algorithmic Model of Affect Learning Style Analytics (NPAMoA-LSA) can be used as a pre-screening instrument for learners to understand better their strengths, weaknesses and preference to reflect their effective learning ability. This tool can also be used for teachers to plan supporting intervention for better teaching pedagogy. Teachers can plan better their teaching materials using relevant teaching aids. Moreover, intervention can be conducted early to facilitate learning and boost the motivation for life-long learning. Hence, the time, effort and monetary budget can be channeled to correct teaching aid to specific learning style individual to get optimal learning experience and exposure (Hamiz et al., 2018).

It is envisaged that the proposed NPAMoA-LSA approach can be embedded in entry test assessment to further understand the strengths, weaknesses and preferences of the learners at preschools. The aspiration of individualized learning through learning analytics is in line with the direction of Ministry of Education to transform Malaysia schools into a learning institution of the future that can sustain to dramatic changes in developing learners holistically.

\section{ACKNOWLEDGEMENT}

The researchers would like to thank Universiti Teknologi MARA (UiTM), International Islamic University Malaysia (IIUM) and Ministry of Higher Education Malaysia (MOHE) for providing financial support through the Transdisciplinary Research Grant Scheme TRGS (TRGS16-04-02-002) to conduct the researcher published in this study.

\section{REFERENCES}

Anonymous, 2016. Quick facts 2016: Malaysia educational statistics. Ministry of Education Malaysia, Kuala Lumpur, Malaysia.

Bhatti, A.M., M. Majid, S.M. Anwar and B. Khan, 2016. Human emotion recognition and analysis in response to audio music using brain signals. Comput. Hum. Behav., 65: 267-275.

Chen, L.C., P. Sandmann, J.D. Thorne, C.S. Herrmann and S. Debener, 2015. Association of concurrent fNIRS and EEG signatures in response to auditory and visual stimuli. Brain Topogr., 28: $710-725$.

Feldman, J., A. Monteserin and A. Amandi, 2015. Automatic detection of learning styles: State of the art. Artif. Intell. Rev., 44: 157-186.

Fleming, N.D. and C. Mills, 1992. Not another inventory, rather a catalyst for reflection. Improve Acad., 11: $137-155$.

Fleming, N.D., 2006. Teaching and Learning Styles: VARK Strategies. 2nd Edn., N.D. Fleming, Christchurch, New Zealand, Pages: 139.

Gevins, A. and M.E. Smith, 2000. Neurophysiological measures of working memory and individual differences in cognitive ability and cognitive style. Cereb. Cortex, 10: 829-839.

Gilakjani, A.P. and S.M. Ahmadi, 2011. The effect of visual, auditory and kinaesthetic learning styles on language teaching. Proceedings of the 2011 International Conference on Social Science and Humanity, February 26-28, 2011, IACSIT Press, Singapore, pp: V2-469-V2-472.

Hamiz, M., M. Bakri, N. Kamaruddin and A. Mohamed, 2018. Assessment analytic theoretical framework based on learners continuous learning improvement. Indonesian J. Electr. Eng. Comput. Sci., 11: $682-687$. 
James, W.B. and D.L. Gardner, 1995. Learning styles: Implications for distance learning. N. Directions Adult Continuing Educ., 1995: 19-31.

Jenke, R., A. Peer and M. Buss, 2014. Feature extraction and selection for emotion recognition from EEG. IEEE. Trans. Affective Comput., 5: 327-339.

Leutner, D., 2014. Motivation and emotion as mediators in multimedia learning. Learn. Instruction, 29: 174-175.

Liew, S.C., J. Sidhu and A. Barua, 2015. The relationship between learning preferences (styles and approaches) and learning outcomes among pre-clinical undergraduate medical students. BMC. Med. Educ., 15: 1-7.
UNESCO., 2015. Education for All 2015: National review report Malaysia. Ministry of Education Malaysia (MOE), UNESCO, Paris, France. https://unesdoc.unesco.org/ark:/48223/pf0000229719

Wang, X.W., D. Nie and B.L. Lu, 2014. Emotional state classification from EEG data using machine learning approach. Neurocomput., 129: 94-106.

Willingham, D.T., E.M. Hughes and D.G. Dobolyi, 2015. The scientific status of learning styles theories. Teach. Psychol., 42: 266-271. 